\section{§42. Stationary Potential Formation and Oscillations in Plasma with Immovable Dust Particles}

Tomita, Y., Smirnov, R. (Grad. Univ. Adv. Studies), Takizuka, T. (Naka Fusion Res. Establishment, JAERI) Zhu, S. (ASIPP, Hefei, China)

Formation of an electrostatic potential due to negatively charged dust particles near a target plate was studied by one-dimensional kinetic analysis. Immovable dust particles are distributed uniformly inside a system. A threshold dust density makes the electric field at the target plate zero. In case of dust density below the threshold a charge-neutral point separates into two between positions of a plasma injection and a wall. An electron sheath near the injection point changes to an ion sheath in front of the target plate through the charge-neutral point. It is clarified that the negatively charged dust particles reduce the ion-sheath potential drop from the neutral point. The stationary oscillation appears in the electrostatic potential for the dust density above the threshold. We confirmed that this oscillation is the DIAW (Dust Ion-Acoustic Wave) with a wavelength of the order of Debye length 1), where dust charges are effective to determine the behavior. The effective dust density to produce the oscillation is as high as $10^{13-14} \mathrm{~m}^{-3}$ for plasma density $\sim 10^{18} \mathrm{~m}^{-3}$ with plasma temperature $\sim 10 \mathrm{eV} .2$ )

The spatial potential distribution is calculated from Poisson equation by using the plasma charge densities and the dust charge density according to the OML theory.

3) The spatial potential profiles for several values of the normalized dust density $n_{d}^{*} R_{d}^{*}\left(=n_{d} R_{d} \lambda_{\text {Des }}^{2}\right.$ ) are shown in Fig.l, where the floating target is located at the position $x_{w}=50 \lambda_{D e s}$, the temperature ratio at the injection point $T_{\mathrm{e}} / T_{\mathrm{j}}$ is 10 , and a hydrogen plasma is considered. Here $n_{\mathrm{d}}$ and $R_{\mathrm{d}}$ are the dust density and radius, respectively, and $\lambda_{\text {Des }}$ is the Debye length at the injection point $(x=0)$. Near the injection point an electron sheath is formed and the middle region of this system a wide quasi-neutral region is formed. In front of the floating target plate an ion-sheath is formed. Note that the wall potential is not disturbed by the immovable dust particles. As the dust density increases, the potential difference between the wall and the position of charge neutrality decreases. As the ion flow velocity $V_{i}$ at the charge-neutral point exceeds the sound speed $c_{s}\left(V_{i} / c_{s} \sim 2.0\right)$ corresponding the Bohm criterion, the potential between the charge-neutral point and the plate monotonically decreases. This potential difference corresponds to the potential drop of the Debye-sheath. As the dust density increases further, the electric field at the wall approaches zero. At the threshold dust density the electric field at the wall becomes zero, whereas the wall potential $\phi_{w}$ still remains the same value. In the case $m_{i} / m_{e}$ $=1836.1$ and $T_{e} / T_{i}=10$, the threshold of the normalized dust density is as high as 0.022 , which corresponds to the dust density as high as $10^{13-14} \mathrm{~m}^{-3}$ for plasma density and temperature of $10^{18} \mathrm{~m}^{-3}$ and $10 \mathrm{eV}$, respectively.

At dust densities above the threshold, a stationary oscillation appears in the potential (Fig.1). The higher dust density, the more the minimal potential, which is the same as the wall potential $\phi_{w}$, is close to the injection point and the deeper potential well becomes. At the further point from the potential minimum $\phi_{w}$, both of the particle fluxes of ions and electrons are the same, which means the floating plate can be located anywhere far from the minimal potential.

In this simple analytical study there are several issues left for the understanding the dust-particle effect in a realistic divertor plasma. The presheath such as the geometric, collisional, ionizing, or magnetic presheath is important to determine the spatial distribution of dust particles. The magnetic field obliquely penetrating the divertor plate affects the potential formation in the existence of dust particles. The Coulomb coupling might be important to investigate dynamics of dust particles in this high dust density. From the results the absorption effect of plasma particles by the dust particles with large radii ( $>$ $1 \mu \mathrm{m}$ ) may not be ignored. For a dust particle with a radius smaller $(<100 \mathrm{~nm})$ the OML theory is not applicable, because of a short Debye shielding length of a dust particle. These effects are needed to incorporate to the model.

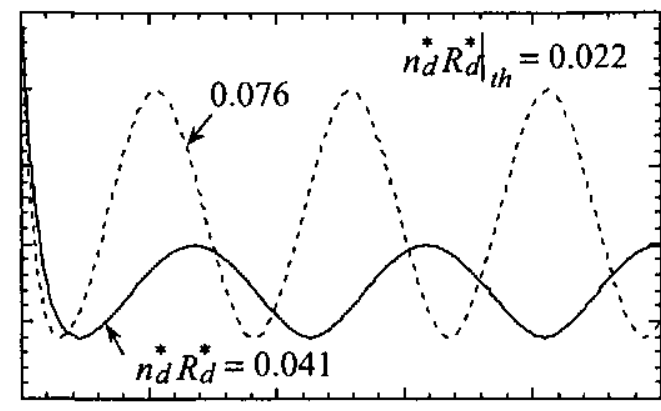

$$
x / \lambda_{\text {Des }}
$$

Fig.1. Spatial distributions of the normalized electrostatic potential for the dust density above the threshold, which is 0.022 .

Acknowledgement

This work is supported by the JSPS-CAS Core-University Program on Plasma and Nuclear Fusion.

References

1) P.K. Shukla and V.P. Silin, Physica Scripta 45 (1992) 508.

2) Y. Tomita , R. Smirnov, et al., to be published in J. Plasma and Fusion Res. SERIES, vol.6 (2004) .

3) J. E. Allen, Physica Scripta, 45 (1992) 497. 